\title{
Auto-afección y espiritualidad. Una filosofía de la religión en Michel Henry ${ }^{1}$
}

\author{
Self affection and spirituality. \\ A philosophy of religion in Michel Henry \\ Olvani F. SÁNCHEZ HERNÁNDEZ \\ Pontificia Universidad Javeriana. Bogotá \\ olvanisan@gmail.com
}

\section{SOBRE LOS PRINCIPIOS DE LA FENOMENOLOGÍA DE LA VIDA}

Michel Henry valora la fenomenología como «la principal corriente de pensamiento de nuestro tiempo $»^{2}$. Sin embargo, considera necesario repensar la empresa fenomenológica originaria y sus desarrollos, pues la fenomenología histórica, al hacer de la intencionalidad su hilo conductor, permanece incapaz de explicitar la esencia de la fenomenalidad pura, tarea específica de la filosofía fenomenológica radical. Ahora bien, escribe Henry, repensar no significa que esta filosofía deba ser solamente «ampliada, corregida, enmendada, ni todavía menos abandonada [...] sino radicalizada de tal modo que se subvierta aquello de lo que todo depende y que, como resultado, en efecto, todo cambie» ${ }^{3}$.

Al establecer la distinción entre lo que aparece y el aparecer mismo, la fenomenología histórica, por supuesto, se preocupó por la determinación de dicho aparecer. Sin embargo, al plantear la pregunta por el aparecer a partir de los fenómenos que aparecen en el mundo, no descubrió otro modo de aparecer que el aparecer del mundo en que tales fenómenos son posibles ${ }^{4}$. De esta forma, piensa Henry, redujo todo aparecer posible al aparecer del mundo y, consecuentemente, «modos de aparecer que abren a formas de experiencia quizá esenciales [en la vida de los hombres] quedaron excluidos a priori por una filosofía que pretendía estar libre de todo supuesto» ${ }^{5}$.

\footnotetext{
${ }^{1}$ El presente texto se origina en el marco de la investigación doctoral que adelanta el autor en la Pontificia Universidad Javeriana de Bogotá, bajo la dirección del profesor Guillermo Hoyos Vásquez.

${ }^{2}$ M. Henry, Fenomenología material, trad. esp., Javier Teira y Roberto Ranz, Madrid, 2009, p. 31.

${ }^{3}$ Idem., p. 32.

${ }^{4}$ Cfr. Idem., p. 46.

${ }^{5}$ Cfr. Idem., p. 46.
} 
Este aparecer en el que mundo y tiempo coinciden en cuanto la temporalidad es la forma de la intencionalidad, ha mostrado su fecundidad en la comprensión de infinidad de objetos; pero resulta insuficiente como principio, como determinación originaria de la esencia de la manifestación. Tal insuficiencia se explica porque, al permitir la diferenciación entre lo que aparece y el aparecer, la intencionalidad que lo funda resulta incapaz de dar cuenta de sí misma y de poner realidad en aquello que hace aparecer. En el horizonte de visibilidad propio de la intencionalidad-mundo-se asegura la salida a la luz de lo manifestado, pero no su existencia que, por el contrario, es afirmada sin una suficiente determinación fenomenológica. Frente a esta insuficiencia, que desmorona la prioridad de la fenomenología sobre la ontología, Henry considera necesario mantener el aparecer como principio para la construcción de una ontología fenomenológica; pero está convencido de que resulta insostenible seguir definiendo todo aparecer concebible, la esencia misma de la manifestación, a partir de las coordenadas del aparecer del mundo. Tal constatación pone en movimiento su radicalización de la fenomenología, bajo la convicción que debe haber un aparecer más originario que el aparecer del mundo, una fenomenalidad capaz de dar cuenta de sí misma, de fundar toda fenomenalidad posible y de constituir el camino de acceso a ella, de ser su propio logos.

De esta forma ve la luz la idea fundante de la fenomenología de la vida: la duplicidad del aparecer. Hay un aparecer originario que no consiste ya en el ek-stasis del mundo; un aparecer inmanente en el que coinciden la manifestación y lo que se manifiesta; un aparecer de lo invisible, de aquello que excluye de sí toda visibilidad. Este aparecer, que constituye la esencia de la manifestación, es comprendido como autorevelación y es denominado vida. La vida es fenomenológica en sentido estricto; la vida no es, adviene ${ }^{6}$; la vida no es una cosa, sino el incesante proceso de auto-revelación, de auto-donación. El aparecer de la vida es, según Henry, el aparecer originario que acontece no desde la lógica de la intencionalidad constitutiva sino desde la dinámica de la donación originaria, de la pasividad radical.

Esta pasividad radical, el cómo de la manifestación originaria, se da como Afectividad primitiva ${ }^{7}$, como auto-afección. Dicha auto-afección refiere, por principio al «ser-afectado, como condición de posibilidad de ser afectado y, en ese sentido, no es ningún contenido de experiencia sino el cómo original de toda manifestación, la materia fenomenológica de su fenomenalidad pura» ${ }^{8}$. El aparecer originario es, pues, auto-afección, auto donación de la vida al viviente que lo constituye como ipseidad pathica, principio de individuación que da al viviente la certeza de estar vivo. Este aparecer originario no intencional, que se funda no en el ek-stasis del ver sino en la inmanencia radical de la afectividad primitiva, es un verdadero fundamento, porque «puede hacerse del ver un problema, pero he aquí lo que carece de significación fenomenológica alguna: que el que experimenta una tristeza, una

\footnotetext{
${ }^{6}$ M. Henry, C'est moi la verité, Paris, 1990, p. 74.

${ }^{7}$ M. Henry, Paroles du Christ, Paris, 2002, p. 99.

${ }^{8}$ M. Henry, Phénoménologie de la vie, T. IV, Paris, 2004, pp. 91, 80.
} 
angustia, una pasión cualquiera, no la experimente o no la experimente tal como la experimenta» ${ }^{9}$.

Ahora bien, ¿cómo acceder, entonces, a la vida si es imposible llegar a ella a partir del aparecer del mundo y de lo que en él aparece? Podemos acceder a la vida sólo por el hecho que ella se nos ha donado y ha hecho de nosotros vivientes, de forma que la vida absoluta y la vida nuestra es una y la misma. Llegamos a la vida llegando a nosotros mismos, experimentándonos a nosotros mismos como condición de posibilidad de todo experimentar ${ }^{10}$. Este logos de la auto-afección, camino hacia la cosa misma de la fenomenología, es denominado por Henry «archi-inteligibilidad de la vida». No se trata de un conocimiento de orden sobrenatural al que debamos «saltar» para conocer la vida, ni de una renuncia a la racionalidad, sino de una inteligibilidad trascendental que precede a todo pensamiento y lo posibilita. No es una inteligibilidad en la que demos cuenta de algo, sino en la que nos experimentamos a nosotros mismos como capaces de «dar cuenta de»y, al tiempo, incapaces de ser la fuente de esa capacidad. La vida no se alcanza en ningún pensamiento, sino en su propia archiinteligibilidad, en la auto-afección que permite distinguir el pensamiento intencional y la revelación del pensamiento mismo ${ }^{11}$.

\section{SOBRE LA COHERENCIA DE LA FENOMENOLOGÍA DE LA VIDA EN SU TEMATIZACIÓN DEL CRISTIANISMO}

En la última década de su trabajo, Henry emprendió un acercamiento fenomenológico al cristianismo, con el ánimo de explicitar la coincidencia descubierta por él entre éste y su teoría fenomenológica ${ }^{12}$. «Cuando trabajaba sobre el cristianismo, [escribe Henry] se produjo para mí un acontecimiento extraordinario; por primera vez mi fenomenología de la vida se encontraba con una fenomenología de la vida y esas dos fenomenologías decían lo mismo» ${ }^{13}$. En la clarificación de esta coincidencia, construyó su filosofía del cristianismo, e indagó por el sentido ontológico, no moral ni espiritual, del contenido del Nuevo Testamento. No estamos ante la pretensión de justificar teóricamente una revelación religiosa, sino ante el intento de develar la inteligibilidad de sus postulados fundamentales a la luz de la «fenomenología de la Vida, cuyos supuestos parecen revelarse como una introducción a las intuiciones decisivas de este movimiento religioso» ${ }^{14}$.

\footnotetext{
${ }^{9}$ M. Henry, Encarnación: una filosofía de la carne, trad. esp., Javier Teira, Gorka Fernández y Roberto Ranz, Salamanca, 2001, p. 95.

${ }^{10}$ Idem., p. 112 ss.

${ }^{11}$ Idem., p. 117.

12 Vid. M. Henry, Le christianisme: une approche phénoménologique, en P. Capelle (ed.) Phénoménologie et christianisme chez Michel Henry, Paris, 2004, pp. 15-32.

13 P. Capelle, Fenomenología francesa actual, Buenos Aires, 2009, p. 58.

14 M. Henry, Incarnation. Une philosophie de la chair, Paris, 2000, p. 317.
} 
Tres preguntas orientan la investigación de la última década de nuestro filósofo, a saber, ¿cuál es el género de verdad del cristianismo ${ }^{15}$, ¿cómo resulta concebible el devenir hombre de dios, en tanto que devenir carne del verbo ${ }^{16}$, y ies posible para el hombre escuchar en su lenguaje una palabra que hablaría en otro lenguaje, el lenguaje de dios? ${ }^{17}$ Como lo afirma el mismo autor, se trata de «cuestiones de principio» ${ }^{18}$ que, de acuerdo con su propósito, mantienen la reflexión en el plano estrictamente filosófico ${ }^{19}$, en cuanto indagan por las pretensiones del cristianismo sin pronunciarse sobre su veracidad. El hecho de recurrir a autores de matriz religiosa y mística, como hace Henry desde sus inicios con el maestro Eckhart, o de tematizar fenomenológicamente los textos del Nuevo Testamento, tal como se lleva a cabo en sus obras finales, no significa la renuncia a la filosofía como pensamiento «desprotegido e indigente [que] se encuentra en su comienzo en una situación errática, sin saber qué es la Verdad ni cómo conducirse para llegar hasta ella. [Un pensamiento que] lejos de estar en posesión de un comienzo seguro de sí mismo, [...] debe salir en busca de un punto de partida verdadero sin saber desde dónde partir para dar con la suerte de su hallazgo, sin saber, en el caso de tener esta suerte, cómo reconocerlo con certeza» ${ }^{20}$.

Esta tematización directa del cristianismo no constituye propiamente un giro en el pensamiento de Henry sino una concentración temática que se entiende en el amplio proyecto de la comprensión de la fenomenalidad ${ }^{21}$. Por demás, si tuviéramos que hablar de un giro, sería preciso tener en cuenta que, como lo sugiere el profesor De Gramont, de existir tal en el pensamiento de un autor, éste sólo es posible a condición de una fidelidad aún mayor ${ }^{22}$. En efecto, los trabajos finales de nuestro autor no se comprenden adecuadamente sin el recurso a los principios de su fenomenología elaborados en la esencia de la manifestación, que actúan como condición de posibilidad en el intento henryano por desentrañar la inteligibilidad filosófica del cristianismo ${ }^{23}$.

\footnotetext{
${ }^{15}$ M. Henry, Yo soy la verdad. Para una filosofia del cristianismo, trad. esp., J. Teira, Salamanca, 2001, p. 9.

${ }^{16}$ M. Henry, Encarnación, op.cit., p. 23.

${ }^{17}$ M. Henry, Paroles du Christ, op.cit., p.13.

${ }^{18}$ Idem.

${ }^{19}$ M. Henry, Encarnación, op.cit., p. 23.

${ }^{20}$ M. Henry, Encarnación, op.cit., p. 327. En este sentido, nos apartamos del diagnosticado giro teológico de la fenomenología cuando con éste se pretende mostrar el abandono del carácter propiamente filosófico de estas fenomenologías, debido a la asunción de una fe religiosa que reposa en el trasfondo de los discursos ( $C f r$. D. Janicaud, La phénoménologie dans tous ses états, Paris, 2009, p. 54). En el seno de la fenomenología ha estado presente, desde los primeros estadios, la posibilidad de formular la pregunta por el-lo absoluto y ha sido, de hecho, formulada y respondida por varios pensadores a quienes no por ello se les acusa de «teólogos anónimos». Para a un recorrido panorámico por los filósofos que se han ocupado de la religión desde la fenomenología, vid., J. Greish, Le buisson ardent et les lumières de la raison. T II, Paris, 2002, pp. 17-375.

${ }^{21}$ M. Henry, Entretiens, op. cit., p. 17.

22 J. De Gramont, La palabra de la vida, Beyruth, 2003, p. 44.

${ }^{23}$ Vid., M. Henry, L'essence de la manifestation, Paris, 1990. Parágrafos 16, 40, 49-51,70. Se desarrollan temas clave como la distinción visible-invisible y la duplicidad del aparecer, la receptividad originaria como esencia del aparecer, la figura de Eckhart y su crítica allogos filosófico desde el significado ontológico de las afirmaciones religiosas, la identificación de la vida absoluta y la vida finita, la consideración legítima de una subjetividad absoluta.
} 
En efecto, en cada uno de los textos que forman la trilogía de su última etapa, se puede identificar claramente el papel de la duplicidad del aparecer y de la auto-afección como dinámica del aparecer originario. A partir de estos principios se establecen las distinciones entre verdad del mundo y verdad de la vida, entre cuerpo y carne, entre palabra del hombre y palabra de Dios, claves de lectura de dichas obras. A partir de estos principios se edifica la noción de hombre como hijo de la vida, carne viviente y oyente de la palabra. A partir de estos principios se adelanta una lectura de la experiencia religiosa, ya no sólo del cristianismo, como segundo nacimiento, como comprensión y dinamización de la relación entre la vida y los vivientes en la carne que somos, en la Afectividad primitiva y sus tonalidades en que es posible escuchar la palabra de la vida, reencontrar la vida que somos.

La «filosofía del cristianismo» esbozada por Henry se edifica, entonces, en relación de coherencia con los presupuestos fundamentales de la fenomenología de la vida. La variación temática no representa un giro en el proyecto filosófico henryano sino una ocasión para evidenciar su fecundidad y ampliar sus desarrollos.

\section{SOBRE LA POSIBILIDAD DE UNA FILOSOFÍA DE LA RELIGIÓN DESDE LA FENOMENOLOGÍA DE LA VIDA}

Para incursionar ahora en el segundo aspecto reconocido por Henry en su pensamiento, la comprensión de problemas y de teorías desde la duplicidad del aparecer ${ }^{24}$, y hacer nosotros mismos el camino, nos preguntamos si es posible elaborar una filosofía de la religión a partir de la fenomenología de la vida. ¿Se puede renovar la comprensión filosófica del fenómeno religioso desde la inversión de la fenomenología emprendida por Michel Henry?25 ¿resulta posible leer toda experiencia religiosa bajo la óptica de la fenomenología de la vida? Si la respuesta es positiva, ¿cuáles serían las notas de esta lectura? Tales serán los interrogantes que orientarán la última parte de nuestro recorrido.

No podemos afirmar que Henry haya hecho explícitamente una filosofía de la religión. Podemos, sin embargo, intentar una lectura de la experiencia religiosa desde los postulados de su fenomenología de la vida. Este intento parece legítimo si tenemos en cuenta que Henry no pretende sentar las bases de una «fenomenología que se encargue de un objeto entre los muchos objetos posibles, sino que pretende esclarecer aquello que constituye el tema específico de la fenomenología, la fenomenalidad misma ${ }^{26}$ en su origen, con la pretensión de que, a partir de ésta, resulten comprensibles, de nuevo, los diferentes objetos en su constitución real, en su estructura ontológica.

\footnotetext{
24 Ver nota 2.

${ }^{25}$ En la formulación de esta pregunta seguimos al profesor J. Greisch en el libro Le buisson ardent et les lumiéres de la raison, T II, Paris, 2002, p.359.

${ }^{26}$ M. Henry, Phénoménologie de la vie, T. IV, Paris, 2004, p.53.
} 
Un segundo elemento que cimienta nuestra pretensión es que estamos ante una fenomenología que, al no encerrar al aparecer en el aparecer del mundo, se muestra ella misma dispuesta a hacerse cargo de «formas de experiencia» moral, estética o religiosa, cuyo sentido y valor suele ser descuidado desde una racionalidad ajustada a los criterios de la verificación y del pragmatismo. «La consideración de ciertos temas religiosos fundamentales [escribe Henry en su último libro] nos permite descubrir un inmenso dominio desconocido del pensamiento llamado racional. Lejos de oponerse a la reflexión verdaderamente libre, el cristianismo [la experiencia religiosa, diríamos nosotros] llevaría a la filosofía tradicional y su cuerpo canónico ante sus límites, por no decir ante su ceguera» ${ }^{27}$.

Para la edificación de este proyecto, dos cuestiones merecen ser consideradas. En primer lugar, es necesario resaltar que Henry considera que la comprensión de la dynamis de la relación entre la vida absoluta y el viviente finito ${ }^{28}$ es el asunto específico de la religión. Relación que es pensada por nuestro fenomenólogo en términos de auto-afección de la vida que da origen a cada ipseidad, (nacimiento trascendental) y de auto-afección del viviente en que tiene lugar el encuentro de cada ipseidad consigo y, allí, el re-encuentro con la vida que es en él y que es él mismo, (segundo nacimiento). En esta dinámica de primer y segundo nacimiento estaría, entonces, la estructura constitutiva de la experiencia religiosa, mejor, experiencia espiritual, que, en consecuencia, podría ser leída como experiencia radical de auto-afección.

En segundo lugar, conviene señalar que para nuestro autor una reflexión es auténticamente filosófica sólo si plantea las preguntas por las condiciones de posibilidad del fenómeno, por sus elementos constitutivos y por su determinación ontológica estructural ${ }^{29}$. De aquí se desprende que una filosofía de la religión que sea tal, no se ocuparía sólo de la descripción de fenómenos catalogados como religiosos ni de la coherencia interna de sus discursos y prácticas, sino que indagará por sus elementos constitutivos y por sus condiciones de posibilidad. Si tenemos en cuenta que «las intuiciones fundamentales de la (una) religión se encuentran en su estructura constitutiva y no es la dispersión de su surgimiento histórico» ${ }^{30}$, lo específico de una filosofía de la religión sería desentrañar la significación ontológica, la comprensión de la relación de la vida con los vivientes, presente en aquello que en la religión es afirmado de forma espontánea. Justamente este es el trabajo que lleva a cabo Michel Henry frente al cristianismo, en diálogo con el Maestro Eckhart. En este sentido, preferimos hablar en adelante más de una filosofía de la espiritualidad que de una filosofía de la religión.

Una filosofía de la espiritualidad, desde la fenomenología de la vida, se ocupará de desentrañar en el seno de las experiencias espirituales, el cómo de la relación entre la vida y el viviente, de tematizar la dynamis de primer y segundo nacimiento. En

\footnotetext{
${ }^{27}$ M. Henry, Paroles du Christ, op. cit., p. 87.

${ }^{28}$ M. Henry, Phénoménologie de la vie, op. cit., p. 54.

${ }^{29}$ M. Henry, Encarnación, op.cit., pp. 110s.

${ }^{30}$ M. Henry, L'essence de la manifestation, Paris, 1990, p. 533.
} 
este marco, la experiencia espiritual puede ser leída como Afectividad, como autoafección del sujeto en la auto-afección de la vida, que engendra un sistema de relaciones que podemos denominar mística y ética.

Este sistema de relaciones define la estructura de la religión misma, y tiene dos notas constitutivas: la inmanencia y la gratuidad. Si tenemos en cuenta que la vida excluye toda estructura ek-stática en su auto-donación, las relaciones que se originan en ella son posibles como relaciones inmanentes, esto es, fundadas en la co-pertenencia de los términos de relación que no se encuentran en un espacio neutro que les es puesto para posibilitar su acercamiento, sino en la dynamis de la vida que es una y múltiple en cada uno de los vivientes y, de este modo, constituye la condición de posibilidad del encuentro entre ellos y entre cada viviente y la vida absoluta ${ }^{31}$.

En cuanto engendrado en la vida, este sistema de relaciones se basa no en el principio de reciprocidad sino en el de la gratuidad, en la lógica de la donación ${ }^{32}$. Mientras la reciprocidad se funda en la posibilidad de reversibilidad de la acción, es decir, que es legítimo esperar del otro en proporción aquello que hemos hecho por él, y viceversa; la gratuidad reconoce que, en tanto vivientes, la relación de la vida para con cada ipseidad es de absoluta donación, imposible de retornar en proporción. Desde la experiencia espiritual así entendida, el hombre se comprende como don, pues, escribe Henry, «nos experimentamos y experimentamos la vida en nosotros como aquello en lo que nosotros vivimos, al mismo tiempo que descubrimos y experimentamos que no nos hemos donado esa vida a nosotros mismos» ${ }^{33}$.

Según hemos dicho, este sistema de relaciones puede ser leído como mística y ética. En lo referente al direccionamiento místico, es preciso retornar a la auto-afección como auto-revelación de la vida originante de cada ipseidad y, al tiempo, como «lugar» del encuentro de la ipseidad consigo misma en el reencuentro con la vida. La experiencia espiritual es experiencia mística por cuanto refiere a esa prueba de sí mismo que descubre a cada ipseidad como don, remitido inevitablemente a la donación que la constituye, a la vida misma que, en su compresión fenomenológica, es auto-donación y no tiene un carácter anónimo. La espiritualidad como mística, desde la fenomenología de la vida, es segundo nacimiento en el que llegamos a nosotros mismos y superamos el «olvido» de la vida.

Ahora bien, comprendida como auto-afección, la experiencia espiritual corresponde a la dinámica misma del vivir humano auténtico. En las tonalidades afectivas en las que tiene lugar la prueba de sí, cada viviente se hace cargo de sí mismo y comienza a comprenderse ya no con relación a las cosas del mundo o con relación a sí mismo en tanto ego constituyente, sino con relación a la vida en la que se descubre

${ }^{31}$ M. Henry, Encarnación, op. cit., p. 314. Desde una fenomenología de la vida, que pone no la exterioridad del mundo sino la auto-revelación de la vida como supuesto del encuentro, se comprenden los «elementos constitutivos» de la intersubjetividad en su «posibilidad trascendental».

32 Dicha intersubjetividad remite no a la interacción de un yo que tiene acceso a los pensamientos de otro yo, sino al encuentro de un viviente que tiene acceso a la vida del otro. En consecuencia, este encuentro es posible gracias no a una razón que se comparte o se proyecta, sino a una conexión vital originariamente afectiva.

${ }^{33}$ M. Henry, Paroles du Christ, op.cit., p. 53. 
constituido. Una experiencia tal puede acontecer en el seno de una tradición religiosa explícita, como lo descubre Henry en el cristianismo, pero ni se reduce a ella ni está asegurada en ella. En tanto experiencia radical de la condición humana, la experiencia mística puede acontecer y acontece en la dinámica misma del vivir y en la multiplicidad de sus esferas.

Hemos dicho también que este sistema de relaciones fundadas en la vida implica necesariamente una comprensión particular del actuar con los demás en tanto que vivientes, una ética. El punto de partida para la relación con el otro no sería el ego constituyente sino la vida en que todos somos constituidos, la vida absoluta que, siendo una y la misma en sí y en cada viviente, es condición de posibilidad del encuentro y la interacción de estos. «Toda relación de un sí con otro sí requiere como punto de partida, no ese Sí mismo, un yo - el mío o el del otro- sino su común posibilidad trascendental, que no es otra cosa que la posibilidad de su relación misma: la Vida absoluta $\rangle^{34}$. Por esto, afirma Henry, «toda comunidad es por esencia religiosa» ${ }^{35}$.

Explicitada así la comunión fundamental con los otros no a partir del mundo en el que estamos sino a partir de la vida que somos, reconocemos que es imposible experimentar al otro, tocarlo, sin tocar la vida que es él mismo. Ni yo constituyo al otro, ni el otro me constituye a mí; ambos nos reconocemos constituidos en la vida, y es este reconocimiento el que funda la auto-donación recíproca entre los vivientes. La espiritualidad como ética, desde la fenomenología de la vida, es dynamis de auto-donación.

\section{BALANCE}

Según hemos intentado mostrar, el proyecto de construir una filosofía de la religión desde la fenomenología de la vida resulta viable cuando se apoya no en la filosofía del cristianismo desarrollada por el autor, sino en los principios fenomenológicos que la hicieron posible y a partir de los cuales resulta comprensible: la duplicidad del aparecer y la auto-afección como esencia del aparecer originario. No se trata de buscar más coincidencias entre esta fenomenología y otras religiones, ni de juzgar la legitimidad de las coincidencias encontradas ya por el autor en su acercamiento al cristianismo. Se trata, mejor, de esbozar unos senderos que permitan desentrañar, a partir de la esencia de la manifestación, de la auto-donación de la vida y de la vida como auto-donación, la determinación ontológica estructural de la experiencia religiosa como auto-afección que devela al sujeto su condición de viviente, lo reencuentra con la vida absoluta y lo remite a los demás vivientes. Por estas razones preferimos usar una categoría quizás más originaria y menos comprometida con los análisis ya realizados desde el ya caracterizado aparecer del mundo, a saber, filosofía de la espiritualidad.

Desde este horizonte, teniendo en cuenta que la historicidad en la que se construyen los diversos testimonios de la experiencia espiritual descansa sobre la donación

\footnotetext{
${ }^{34}$ Idem., p. 49.

${ }^{35}$ M. Henry, Encarnación, op.cit., p. 315.
} 
originaria de la vida, para comprender filosóficamente dichas experiencias se puede y se debe enfrentar la necesidad de establecer entre el mundo y la vida una relación que no sea la de exclusión mutua ${ }^{36}$. Es preciso establecer la relación entre la auto-afección, entraña de la experiencia espiritual, y los diferentes lenguajes en que se intenta expresarla. Es cierto que no se puede hacer fenomenología de la religión sin tener en cuenta el carácter histórico de las religiones (Ricoeur) ${ }^{37}$; pero conviene no perder de vista, tampoco, que una auténtica fenomenología radical de la religión se hace cargo de su estructura constitutiva y no en la dispersión histórico-mundana de su surgimiento y configuración (Henry) ${ }^{38}$. Resulta imperioso tender puentes entre la percepción, el lenguaje y la auto-afección, de modo que podamos hacernos cargo de las experiencias religiosas en que se actualiza la donación de la vida absoluta a los vivientes finitos, desentrañar su significación última sin destruirlas, o estaremos condenados al mutismo sobre éstas y otras formas de experiencia constitutivas de la condición humana.

${ }^{36}$ Idem., p. 317.

${ }^{37}$ M. Henry, Yo soy la verdad, op. cit., p. 53.

${ }^{38}$ P. Ricœur, Phénoménologie et théologie, Paris, 1992, pp. 15-40.

${ }^{39}$ M. Henry, L'essence de la manifestation, op.cit., p. 533. 